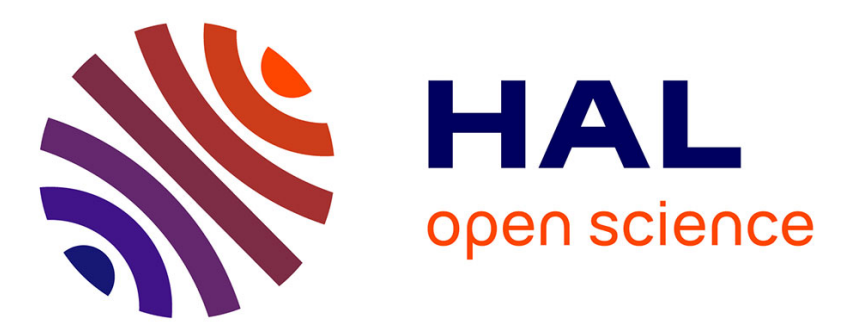

\title{
Oscillatory instability of the gas-liquid meniscus in a capillary under the imposed temperature difference
}

\author{
Vadim Nikolayev
}

\section{To cite this version:}

Vadim Nikolayev. Oscillatory instability of the gas-liquid meniscus in a capillary under the imposed temperature difference. International Journal of Heat and Mass Transfer, 2013, 64, pp.313 - 321. 10.1016/j.ijheatmasstransfer.2013.04.043 . cea-01481073

\section{HAL Id: cea-01481073 https://hal-cea.archives-ouvertes.fr/cea-01481073}

Submitted on 2 Mar 2017

HAL is a multi-disciplinary open access archive for the deposit and dissemination of scientific research documents, whether they are published or not. The documents may come from teaching and research institutions in France or abroad, or from public or private research centers.
L'archive ouverte pluridisciplinaire HAL, est destinée au dépôt et à la diffusion de documents scientifiques de niveau recherche, publiés ou non, émanant des établissements d'enseignement et de recherche français ou étrangers, des laboratoires publics ou privés. 


\title{
Oscillatory instability of the gas-liquid meniscus in a capillary under the imposed temperature difference
}

\author{
Vadim S. Nikolayev* \\ ESEME, Service des Basses Températures, UMR-E CEA/UJF-Grenoble 1, INAC, Grenoble, France \\ ESEME, PMMH-ESPCI, 10 rue Vauquelin, 75231 Paris Cedex 5, France
}

\section{A R T I C L E I N F O}

\section{Article history:}

Received 9 January 2013

Received in revised form 4 April 2013

Accepted 15 April 2013

Available online 15 May 2013

\section{Keywords:}

Evaporation

Gas-liquid meniscus

Oscillations

Instability

Pulsating heat pipe

\begin{abstract}
A B S T R A C T
To understand the oscillations in the real, multi-branch pulsating heat pipe (PHP), the start-up conditions of the single branch PHP with no adiabatic section are studied theoretically and numerically. The single branch PHP is a capillary open from one end, which is connected to a reservoir at constant pressure. A gas bubble is confined between the sealed end of the capillary and the liquid. The gas is the vapor of the liquid. The gas end of the capillary is maintained at a constant temperature larger than that of the liquid end. Under certain conditions, self-sustained oscillations of the meniscus may exist in such a system. The conditions of oscillation development (i.e. of the PHP startup) are obtained via the stability analysis of an earlier proposed theoretical model. The linear instability is absent in such a system. The instability of a marginal state described by piece-wise linear equations is analyzed with the analytical averaging method. The instability boundary is presented in terms of dimensionless groups, the physical significance of which is discussed. It is found that the model describes correctly the known experimental facts. Some predictions concerning the instability threshold are formulated.
\end{abstract}

(c) 2013 Elsevier Ltd. All rights reserved.

\section{Introduction}

Cooling of devices like microprocessors or engines of transportation vehicles is extremely important for their functioning. In most cases it is vital for their reliability and energy efficiency. A growth of their power during the last decades stimulates research of new heat transfer solutions. The passive heat transfer devices called heat pipes are used more and more widely as thermal links connecting the heat sources to colder radiators. The conventional heat pipe is a sealed tube partially filled with a fluid. The fluid is vaporized in the portion (or section) of the tube called evaporator and recondensed back in the condenser (i.e. cold) section. The energy is thus stored in the evaporator as the latent heat and released in the condenser. There are several kinds of heat pipes that differ mainly by the mechanism of liquid transport (gravitational convection, capillary suction, etc.) from the condenser to evaporator.

The pulsating (or oscillating) heat pipe (PHP) is a long capillary tube bent into many branches and partially filled with a twophase, usually single component, working fluid [1]. The tube is of simple circular cross-section. The branches meander between the hot and cold areas thus forming multiple evaporator and condenser sections. During PHP functioning, a pattern of multiple va-

\footnotetext{
* Address: ESEME, PMMH-ESPCI, 10 rue Vauquelin, 75231 Paris Cedex 5, France. Tel.: +331407958 26; fax: +33140794523.

E-mail address: vadim.nikolayev@espci.fr
}

por bubbles separated by liquid plugs forms spontaneously inside the tube. Unlike the other types of the heat pipes, the functioning of PHP is intrinsically non-stationary. The evaporation/condensation triggers self-sustained oscillations of the bubble-plug structure. These oscillations are very important because they lead to a generally substantially lower thermal resistance in comparison with other types of heat pipes. In addition to the latent heat mechanism, the heat is transferred in PHP via the sensible heat transfer when the colder liquid plugs come in contact with the condenser or hotter liquid plugs sweep the evaporator.

Because of their simplicity and high performance, PHPs are often considered as highly promising. Their industrial application is however limited because the functioning of PHPs is not completely understood. The reliability of heat pipes is however a critical issue because a sudden halt of functioning (halt of oscillations in case of PHP) can lead to the meltdown of the cooled industrial device.

During the last decade, researchers have extensively studied PHPs [2,3]. A large number of hydrodynamic and microscopic phenomena are involved in their functioning [4]. The main flow pattern inside the PHP is the slug flow, i.e. the flow of the "Taylor bubbles" where the gas is surrounded by the thin liquid film deposed on the internal tube walls by the receding menisci. For isothermal systems (with no heating or cooling), the film is continuous within a bubble. Its thickness depends on the meniscus velocity and is usually micrometric [5,6]. The physics of the film formation (and its thickness scaling) is similar to the 


\begin{tabular}{|c|c|c|c|}
\hline \multicolumn{4}{|c|}{ Nomenclature } \\
\hline C & variable defined by Eq. (A.5) & $\gamma$ & adiabatic index \\
\hline$c$ & specific heat $[\mathrm{J} /(\mathrm{kg} \cdot \mathrm{K})]$ & \multicolumn{2}{|c|}{$\kappa, \xi, \varphi, \psi$ dimensionless phases } \\
\hline $\mathrm{Ca}$ & capillary number & $\mu$ & liquid shear viscosity $[\mathrm{Pa} \cdot \mathrm{s}]$ \\
\hline$D$ & variable defined by Eq. (A.4) & $v$ & liquid kinematic viscosity $\left[\mathrm{m}^{2} / \mathrm{s}\right]$ \\
\hline$d$ & tube diameter $[\mathrm{m}]$ & $\rho$ & liquid density $\left[\mathrm{kg} / \mathrm{m}^{3}\right]$ \\
\hline$F$ & force $[N]$ & $\sigma$ & surface tension $[\mathrm{N} / \mathrm{m}]$ \\
\hline$h_{l v}$ & latent heat $[\mathrm{J} / \mathrm{kg}]$ & $\tau$ & characteristic time scale [s] \\
\hline$k$ & heat conductivity $[\mathrm{W} /(\mathrm{m} \cdot \mathrm{K})]$ & & \\
\hline$L$ & length $[\mathrm{m}]$ & \multicolumn{2}{|c|}{ Subscripts and superscripts } \\
\hline$m$ & mass $[\mathrm{kg}]$ & $c$ & condenser \\
\hline$p$ & pressure $[\mathrm{Pa}]$ & $d$ & dry part of evaporator \\
\hline$q_{\text {sens }}$ & sensible heat power $[\mathrm{W}]$ & $e$ & evaporator \\
\hline$R$ & vapor gas constant $[\mathrm{J} /(\mathrm{kg} \cdot \mathrm{K})]$ & $f$ & friction, film \\
\hline$r$ & dimensionless velocity amplitude & $g$ & gas \\
\hline$S$ & tube cross-section area $\left[\mathrm{m}^{2}\right]$ & $i$ & either $e$ or $c$ \\
\hline$T$ & temperature $[\mathrm{K}]$ & $l$ & liquid \\
\hline$t$ & time $[\mathrm{s}]$ & $m$ & meniscus \\
\hline$U$ & heat transfer coefficient $\left[\mathrm{W} /\left(\mathrm{K} \cdot \mathrm{m}^{2}\right)\right]$ & $r$ & reservoir \\
\hline$v$ & meniscus velocity $[\mathrm{m} / \mathrm{s}]$ & sat & saturation \\
\hline \multirow[t]{2}{*}{$x$} & meniscus position $[\mathrm{m}]$ & $t$ & total \\
\hline & & $V$ & at constant volume \\
\hline \multicolumn{2}{|c|}{ Greek symbols } & 0 & at $t=0$ \\
\hline $\begin{array}{l}\alpha, \beta, \\
\delta\end{array}$ & $\begin{array}{l}\text { coefficients defined by Eq. (22) } \\
\text { thickness [m] }\end{array}$ & & \\
\hline
\end{tabular}

Landau-Levich phenomenon [7]. While the Taylor bubbles are being extensively investigated in the isothermal situation, their experimental studies for the cases where the phase change is involved are rare. In the single known to me experimental study, Lagubeau [8] observed the successive events of the film deposition by the receding meniscus and the film dryout in such a way that the film length varied during the meniscus oscillations.

There are several modeling approaches available in the literature. Shafii et al. [9] pioneered the modeling approaches for multi-branch PHPs with a one-dimensional (1D) model. The gas-liquid menisci were flat and the evaporation-condensation mass exchange was accounted for by the temperature difference terms $\propto\left(T_{\text {wall }}-T\right)$ where $T$ is the vapor temperature and $T_{\text {wall }}$ was either $T_{e}$ or $T_{c}$ depending on the bubble location. The temperatures of the evaporator $T_{e}$ and condenser $T_{c}$ were fixed. The film existence was neglected. The vapor was assumed to obey the ideal gas equation of state. Periodical (nearly sinusoidal) oscillations appeared after a transient. Contrary to experimental observations, their amplitude was small with respect to the sizes of the condenser or the evaporator.

It is well known from the analysis of conventional heat pipes that most of evaporation in the evaporator occurs through the liquid films. To account for this effect, the above model was extended later by the same group to the 3D axisymmetric case with the static liquid films with spatially varying thickness [10]. The mass exchange was however modeled similarly to the previous case and the model showed a similar behavior. Dobson [11] introduced a lumped meniscus geometry (Fig. 1) which allowed him the variable film account within a $1 \mathrm{D}$ model. The liquid film was assumed to always cover the inner walls of the tube (i.e. to be continuous) in the condenser when a part of the bubble was located inside it. The film was allowed to dry out partially or completely in the evaporator. Apart from the film introduction, the model was similar to its predecessors. Single branch PHP (Fig. 1) was considered. It is a straight capillary with a sealed end, which is heated (evaporator). The gas bubble is confined between the sealed end and a liquid plug. The condenser end of the capillary is connected to a large reservoir filled partially with the liquid at constant pressure $p_{r}$.
Das et al. [12] performed the instability analysis with the model of Shafii et al. [9] applied to the single branch PHP and have concluded that the model was not adequate. The regime of self-sustained oscillations during which the meniscus penetrated into both the condenser and evaporator have not been found. The instability (i.e the oscillatory growth of a small initial perturbation) occurred only when the meniscus was located in the condenser, without its penetration into the evaporator. To provide an adequate description, Das et al. [12] have introduced the "film evaporation-condensation"(FEC) model. It is a $1 \mathrm{D}$ model using the lumped meniscus geometry. The heat/mass exchange is described by the terms $\propto\left(T_{\text {wall }}-T_{\text {sat }}\right)$, where $T_{\text {sat }}$ is calculated for the current gas pressure $p$. The FEC model describes large amplitude oscillations during which the meniscus sweeps both the condenser and the evaporator. The FEC model agrees quantitatively with the experimental results [12] on the single branch PHP. Recently, the FEC model has been validated against the data obtained with another experimental set-up [13]. It described most features observed experimentally (like the intermittency of oscillations observed by both Das et al. [12] and Rao et al. [13]). The FEC model was applied with success to the single branch PHP functioning with cryogenic fluids [14] and was shown to reproduce correctly the dependence on the PHP inclination angle. It was applied also

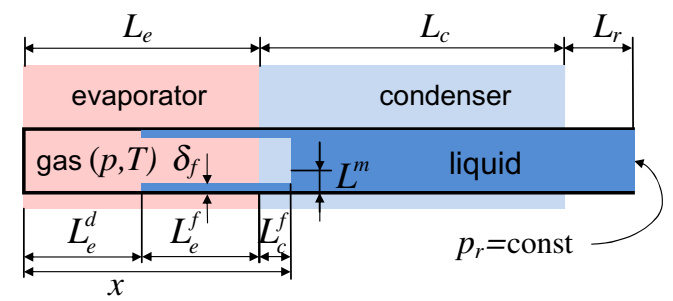

Fig. 1. Single branch PHP within the lumped meniscus approximation. The total tube length $L_{t}=L_{e}+L_{c}+L_{r}$ includes an effective length $L_{r}$ representing an amount of the liquid in the reservoir that takes part in the oscillating motion; $L_{e}$ and $L_{c}$ are the lengths of the respective tube sections. 
to simulate the multi-branch PHP $[15,16]$. These simulations were shown to reproduce at least qualitatively the main experimentally observed features of the multi-branch PHP functioning.

While the FEC model is successful in the numerical description of the PHP, the simulations cannot give an understanding of the startup criteria for the oscillations or explain the origin of the instability that causes them. The aim of this work is to analyze the instability for the simplest case of the single branch PHP with no adiabatic section (Fig. 1). Due to simplicity of the FEC model, an analytical approach is possible.

The article is structured as follows. The model [12] is summarized in Section 2. The equilibrium state for such a system is identified in Section 3. The dimensionless groups of parameters that rule out the oscillation startup are discussed in Section 5. The stability of the equilibrium state with respect to small perturbations is studied in Section 6. The equilibrium state turns out to be marginal, and the conventional linear analysis of its stability is thus impossible. The latter is performed with the time averaging method (called sometimes Krylov-Bogoliubov method). The results are summarized in Section 7.

\section{Film evaporation-condensation model}

The lumped meniscus geometry (Fig. 1) is an approximation of the curved meniscus to reduce the dimensionality of the model. The film thickness is assumed to be constant while the film length in the evaporator may vary in the agreement with the above cited experiments. Such a choice can also be justified by the strong rate of evaporation in the vicinity of the triple vapor-liquid-solid contact line [17] and the capillary forces that both tend to reduce the film length and lead to the partial drying of the evaporator [18]. As a film thickness guess, one may use a the data fit [6] obtained for the isothermal case. According to it, the film thickness is

$\delta_{f}=d \frac{0.67 C a^{2 / 3}}{1+3.35 C a^{2 / 3}}$

where $d$ is the tube diameter. The numerator of Eq. (1) coincides with the Bretherton [5] formula. The capillary number $C a=\mu v_{R M S}$ / $\sigma$ may be based on the root mean square meniscus velocity $v_{R M S}$. In what follows, $\delta_{f}$ will be considered a parameter.

It is assumed that in the absence of the evaporation/condensation, the film edge is pinned and does not move. The length of the dry part of evaporator $L_{e}^{d}$ (Fig. 1 ) thus obeys the equation

$\dot{L}_{e}^{d}= \begin{cases}v & \text { if } L_{e}^{d} \geqslant x, \quad v<0, \\ \dot{m}_{e}^{f} /\left(\rho \pi d \delta_{f}\right) & \text { otherwise, }\end{cases}$

where $\dot{m}_{e}^{f}$ is the film evaporation rate in the evaporator and

$v=\dot{x}$.

The first line of Eq. (2) corresponds to the meniscus advancing over the dry evaporator (where $L_{e}^{d}=x$ ) and the second corresponds to the effect of evaporation. In principle, $L_{e}^{d} \leqslant x$ so that the condition $L_{e}^{d}>x$ may seem to be unnecessary. It is however useful when the solution of Eq. (2) becomes slightly larger than $x$ in a numerical calculation (because of the finiteness of the time step) or in the approximate analytical approach considered below.

The vapor mass exchange is assumed to be limited by the heat conduction in the film [10]. This leads to the following heat balance equations at the film-gas interface in evaporator $(i=e)$ or condenser $(i=c)$ :

$h_{l v} \dot{m}_{i}^{f}=U \pi d L_{i}^{f}\left[T_{i}-T_{s a t}(p)\right]$.

Within a factor $\lesssim 1$ accounting for the spatial variation of the film thickness, $U \sim k_{l} / \delta_{f}$. Eq. (4) means that the heat flux that comes from the liquid side of the film is spent to vaporize the liquid. The residual heat flux to the vapor is neglected with respect to the liquid heat flux.

The liquid film is continuous in the condenser. The film length is thus equal to the length of the bubble in it (Fig. 1),

$L_{c}^{f}= \begin{cases}0, & \text { if } x \leqslant L_{e}, \\ x-L_{e}, & \text { if } L_{e}<x \leqslant L_{e}+L_{c}, \\ L_{c}, & \text { otherwise. }\end{cases}$

The film length in the evaporator is defined with $L_{e}^{d}$

$L_{e}^{f}= \begin{cases}x-L_{e}^{d}, & \text { if } \quad L_{e}^{d}<x<L_{e}, \\ 0, & \text { if } x \leqslant L_{e}^{d}, \\ L_{e}-L_{e}^{d}, & \text { if } \quad x \geqslant L_{e} .\end{cases}$

Note that the governing differential equation is written for $L_{e}^{d} ; L_{e}^{f}$ is defined through $L_{e}^{d}$. This is different from the original model [12], where the equation was written for $L_{e}^{f} ; L_{e}^{d}$ was defined with $L_{e}^{f}$. While both approaches are equivalent, such a change is necessary to make the amplitude of oscillations of main variable smaller than its average value (which is necessary for the averaging method used below). The latter criterion usually holds for $L_{e}^{d}$ and breaks down for $L_{e}^{f}$.

A thermal boundary layer is allowed to exist in the vapor, so that the temperature $T$ of its bulk may be different from $T_{\text {sat }}(p)$. This is possible due to the smallness of heat diffusion in the gas. The gas obeys the ideal gas equation

$p=\frac{m R T}{S x}$.

Although much weaker than at the film interface, evaporation and condensation might occur at the remaining meniscus part (other than the film) and may be of some importance if the film is evaporated completely. The heat balance on the meniscus depends on whether the meniscus situates inside the evaporator $(i=e)$ or the condenser $(i=c)$ :

$h_{l v} \dot{m}_{i}^{m}=U^{m} \pi d L_{i}^{m}\left[T_{i}-T_{s a t}(p)\right]$.

The lengths that enter the above equations are defined so as to account for the exact location of the meniscus,

$L_{e}^{m}= \begin{cases}L^{m}, & \text { if } x<L_{e}, \\ 0, & \text { otherwise },\end{cases}$

$L_{c}^{m}= \begin{cases}L^{m}, & \text { if } L_{e}<x \leqslant L_{e}+L_{c}, \\ 0, & \text { otherwise }\end{cases}$

where $L^{m}$ is a length of the portion of the meniscus on which the mass exchange occurs (cf. Fig. 1) and $U^{m}$ is the corresponding heat exchange coefficient. The vapor mass variation includes several contributions described above,

$\dot{m}=\dot{m}_{e}^{f}+\dot{m}_{c}^{f}+\dot{m}_{e}^{m}+\dot{m}_{c}^{m}$.

Since the terms $\dot{m}_{i}^{m}$ are generally much smaller than the corresponding film-related quantities, their influence on the instability criterion will be neglected; the meniscus evaporation is however important for the determination of equilibrium states.

The vapor heat balance [19] reads

$m c_{V} \dot{T}=\dot{m} R T+q_{\text {sens }}-p S v$.

The sensible heat exchange of the tube with the vapor in the evaporator is accounted for by the term

$q_{\text {sens }}=U_{g} \pi d L_{e}^{d}\left(T_{e}-T\right)$.

The heat exchange coefficient $U_{g}$ is proportional to the vapor heat conductivity $k_{g}$. Because of its smallness, the sensible heat exchange with the gas is much weaker than the exchange by 
evaporation/condensation. Its impact on the oscillations will be discussed below. The momentum equation for the liquid plug reads

$\frac{\mathrm{d}\left(v m_{l}\right)}{\mathrm{d} t}=\left(p-p_{r}\right) S-F_{f}$,

where

$m_{l}=\rho\left(L_{t}-x\right) S$

is the liquid mass. The friction force $F_{f}$ accounts for the liquid single phase contribution and is proportional to $v^{2}[12]$. For this reason it does not influence the linear stability conditions and will be omitted hereafter. It is likely that the viscous friction appearing because of the presence of the liquid films is proportional to $v$. Since the film contribution to $F_{f}$ is not yet identified (to my best knowledge), it will be neglected.

It is important to note that the parameters need to be chosen in such a way that

$T_{c}<T_{\text {sat }}\left(p_{r}\right)<T_{e}$

to allow for the mass exchange both in condenser and evaporator.

Five governing ordinary differential Eqs. 2, 3, 9, 10 and 12 of the model are now defined. They need to be solved to find the temporal evolution of the PHP.

\section{Equilibrium states}

First one needs to identify the equilibrium states, the stability of which will be analyzed next. They can be found by putting to zero the time derivatives. One deduces from Eqs. 3, 4, 11 and 12 the respective conditions

$\bar{v}=0, \quad \bar{L}_{e}^{f}=0, \quad \bar{T}=T_{e}, \quad \bar{p}=p_{r}$

that should hold for any equilibrium state. The equilibrium values are denoted here by the bar.

The occurrence of equilibrium states depends on the tube section to which the meniscus belongs. It is evident that two cases where the meniscus belongs to the condenser and the evaporator are not symmetrical: in either case there is a part of the bubble that belongs to the evaporator. One mentions that under the conditions (14) and (15), no equilibrium is possible when the meniscus is located in the condenser, i.e. at $\bar{x}>L_{e}$. Indeed, according to Eqs. (4) and (5), the condensation always takes place in this case. The gas volume decreases and the meniscus displaces toward the evaporator. Similarly, when the meniscus is located in the evaporator, the equilibrium is impossible because of the (weak) evaporation from the meniscus, cf. Eq. (8). The evaporation causes a displacement of the meniscus toward the condenser. It appears that a unique equilibrium state corresponds to the meniscus location exactly at the boundary between the evaporator and the condenser $\left(\bar{x}=\bar{L}_{e}^{d}=L_{e}\right)$. This feature is in the complete coherence with the experiment and the common sense. However, it is completely ignored in the "superheated vapor model" [9]. Indeed, Das et al. [12] have shown that the equilibrium is achieved within this model when the meniscus situates anywhere inside condenser or evaporator.

\section{Characteristic time scale}

The characteristic time scale $\tau$ is related to the eigenfrequency of oscillations in the absence of mass transfer and energy dissipation. $\tau$ can be found by considering small deviations (denoted by $\Delta$ ) from equilibrium, e.g. $x=\bar{x}+\Delta x$. By linearizing Eq. (7), one obtains

$\frac{\Delta p}{\bar{p}}=\frac{\Delta m}{\bar{m}}+\frac{\Delta T}{\bar{T}}-\frac{\Delta x}{\bar{x}}$, where $\Delta m=0$ needs to be put because of the constraint of the absence of mass transfer. From (7) and (15) one gets

$\bar{m}=\frac{p_{r} S L_{e}}{R T_{e}}$.

From the set containing Eq. (3) and equations

$\bar{m} c_{V} \Delta \dot{T}=-\bar{p} S v$

$\bar{m}_{l} \dot{v}=\Delta p S$,

one deduces the eigenfrequency $\omega=1 / \tau$,

$\tau=\sqrt{\frac{\rho \bar{x}\left(L_{t}-\bar{x}\right)}{p_{r} \gamma}}$,

where $\gamma=\left(c_{V}+R\right) / c_{V}>1$ is the adiabatic index. Its presence points out to the effect of the adiabatic heating/cooling of the vapor during its compression/expansion, respectively. If the gas was assumed isothermal, only Eqs. (3) and (19)) would need to be solved together. The resulting expression [8] for $\tau$ would differ from Eq. (20) by the absence of the factor $\gamma$.

\section{Linearization}

The scale $\tau$ can now be used to make the time dimensionless. $\bar{x}$ is used to make the lengths dimensionless. The velocity scale is thus $\bar{x} / \tau$. The equilibrium values (15) are used to make the remaining deviations dimensionless. The tilde signifies the respective dimensionless deviation, e.g. $\tilde{x}=\Delta x / \bar{x}$. The indexes of $\widetilde{L}_{e}^{d}$ will be dropped hereafter for simplicity. The reduced, dimensionless, and linearized governing equations may be defined as follows. Eqs. (3) and (10) read

$\dot{\tilde{x}}=\tilde{v}$

$\dot{\widetilde{T}}=(\gamma-1)(\dot{\tilde{m}}-\tilde{v})-\varepsilon \widetilde{T}$.

By using Eq. (16) in Eq. (12), one obtains

$\gamma \dot{\tilde{v}}=\widetilde{T}-\tilde{x}+\tilde{m}$.

Eqs. (2) and (9) read, respectively,

$\dot{\tilde{L}}= \begin{cases}\tilde{v}, & \text { if } \tilde{L} \geqslant \tilde{x}, \quad \tilde{v}<0, \\ \left.\dot{\tilde{m}}\right|_{\alpha_{c} \rightarrow 0} / \beta, & \text { otherwise, }\end{cases}$

$\dot{\tilde{m}}=\beta \begin{cases}0, & \text { if } \quad \tilde{x} \leqslant \tilde{L}, \\ \alpha_{e}(\tilde{x}-\widetilde{L}), & \text { if } \quad \tilde{L}<\tilde{x}<0, \\ -\alpha_{e} \widetilde{L}-\alpha_{c} \tilde{x}, & \text { if } \quad \tilde{x} \geqslant 0,\end{cases}$

where the negligibly small contributions of the meniscus evaporation/condensation are dropped as discussed above. Apart from the adiabatic index $\gamma$, there are four dimensionless numbers that affect the stability of the system. The first of them

$\varepsilon=\frac{U_{g} \pi d L_{e} \tau}{\bar{m} c_{V}}$

is the ratio of $\tau$ and the time scale that corresponds to the heat exchange of the tube with the gas. Two following numbers characterize the film condensation in the condenser and film evaporation in the evaporator, respectively:

$\alpha_{c}=\frac{U \tau\left[T_{s a t}\left(p_{r}\right)-T_{c}\right]}{\rho \delta_{f} h_{l v}}$,

$\alpha_{e}=\frac{U \tau\left[T_{e}-T_{s a t}\left(p_{r}\right)\right]}{\rho \delta_{f} h_{l v}}$. 
They are the ratios of $\tau$ and the respective time scales. The last number

$\beta=\frac{\pi d \delta_{f} L_{e} \rho}{\bar{m}}$.

is the ratio of the film mass and the gas bubble mass provided they are of the same length. Note that since $\bar{m} \propto L_{e}$ (cf. Eq. (16)), neither of these parameters depends explicitly on $L_{e}$. The $L_{e}$ dependence of $\alpha_{i}$ occurs only through $\tau$.

The stability of the set of Eq. (21) may be analyzed now.

\section{Stability analysis}

Eq. (21) are not linear (they are piecewise linear, PWL) and thus the stability of the system cannot be obtained with the classical linear stability analysis. The averaging method [20] applied usually to the nonlinear systems can be used. It consists in the identification of slowly varying (in comparison with the oscillation frequency) variables and the averaging over the remaining "fast" variables.

The numerical solutions of the rigorous PWL Eq. (21) can now be compared to the solutions given by the averaging method presented in Appendix A, see Fig. 2. The initial conditions

$\tilde{m}=0, \quad \tilde{v}=r_{0}, \quad \widetilde{T}=0, \quad \tilde{x}=0, \quad \widetilde{L}=0$,

where $r_{0} \ll 1$, are used.

Fig. 2 shows that the meniscus oscillates around an average position defined by the reduced vapor mass. The average position given by the PWL equations fluctuates but its fluctuations are small, which provides the justification for the averaging approximation. The film edge $\widetilde{L}$ shows a similar behavior. The averaged description of the film length is however worse because its initial strong variation is smoothed. A better agreement may probably be attained in the next order of approximation where both the mean value and the average amplitude of the film edge position are introduced as slow variables (only the first of them was considered here). The growth rate of the amplitude of oscillations is underestimated by the averaging approximation (Fig. 2b). The approximation quality declines with the increase of growth rate.

Depending on the system parameters, the oscillations may decline (Fig. 2(a)) to the equilibrium state or, on the contrary, grow (Fig. 2(b)). The latter situation corresponds to the instability. A pri-

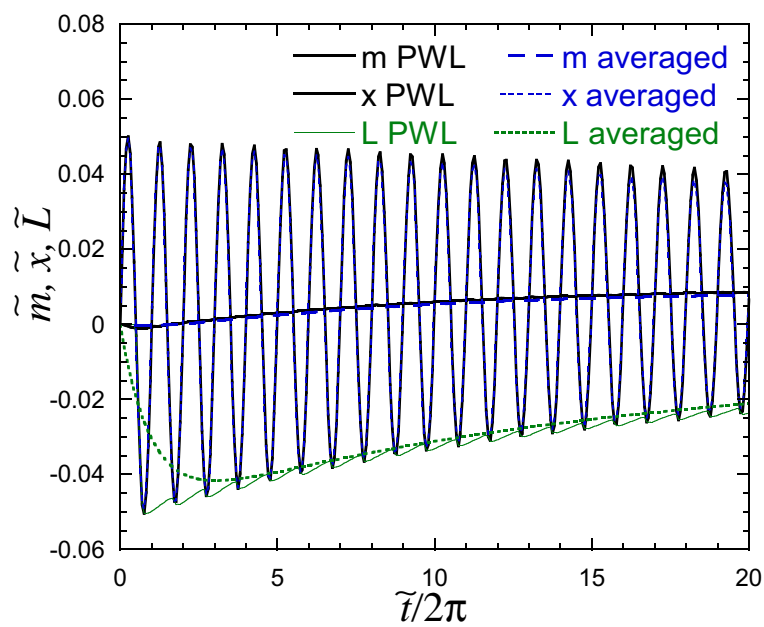

(a) ori, the averaging approximation should be suitable for the instability threshold determination because the oscillation amplitude (which is one of the slow variables) does not vary when the parameters correspond to the threshold. To find the instability criterion, one needs to analyze the behavior of the oscillation amplitude at large times, see Appendix B.

Eqs. (B.5) define the instability threshold within the averaging approximation. The threshold may be expressed as a dependence of the dimensionless group $(\gamma-1) \varepsilon / \beta$ on $\alpha_{c}$ and $\alpha_{e}$. This function is shown in Fig. 3. The system is unstable for the parameters that correspond to the points below the surface and stable for the points above the surface.

Several general conclusions can be made from this result. First, both condensation and evaporation are necessary for oscillations. Indeed, if either $\alpha_{c}$ or $\alpha_{e}$ are zero, no instability is possible. The increase of $\alpha_{c}$ or $\alpha_{e}$ favors the instability. If the system is characterized by a point located above the surface in Fig. 3 (i.e. in the stable region), an appropriate increase of $\alpha_{c}$ and $\alpha_{e}$ (achieved e.g. by increasing the temperature difference $T_{e}-T_{c}$ ) brings the system to the instability. This agrees with the experiment where a threshold value of the above temperature difference is one of few well established features of the PHP startup [2,12].

Another consequence of Fig. 3 is that the heat exchange of the dry evaporator walls with the gas (characterized by $\varepsilon$ ) is the energy dissipation and hinders the oscillations. Indeed, an increase of $\varepsilon$ may bring the system above the surface in Fig. 3, where the system is stable. This feature can be easily understood. Since the heat exchange between the dry evaporator walls and the gas does not contribute to the evaporation/condensation mass exchange (which is the moving force of oscillations), the corresponding energy is lost, which means that $\varepsilon$ characterizes the energy dissipation. Since $\varepsilon \sim U_{g} \sim k_{g}$, the oscillation threshold in terms of $T_{e}-T_{c}$ should be lower for the fluids with lower $k_{g}$. This means that, in general, the fluids with low gas heat conductivity are advantageous for the PHP.

The impact of the film thickness on the stability is more sophisticated. On one hand, an increase of $\delta_{f}$ leads to the decrease of $\alpha_{c}$ and $\alpha_{e}$ and thus hinders the instability. On the other, it leads to the increase of $\beta$ which favors the instability. This means that in general there is a range of $\delta_{f}$, for which the oscillations develop. Beyond this range the system is stable.

The rigorous instability threshold can be found by solving numerically Eq. (21), comparing the velocity amplitude at long

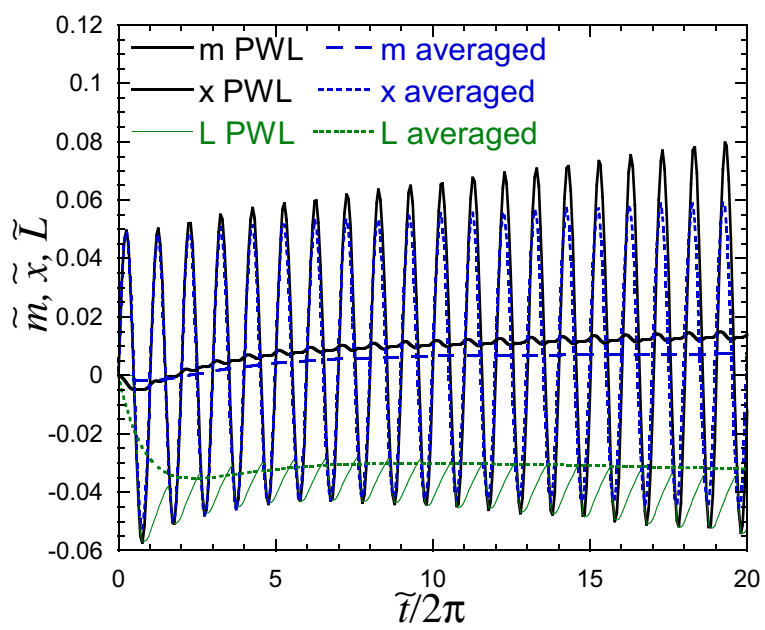

(b)

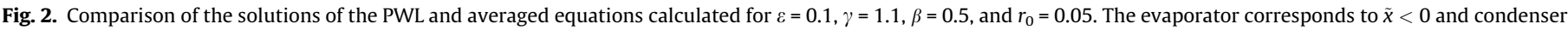
to $\tilde{x}>0$. (a) Below instability threshold; $\alpha_{e}=0.02, \alpha_{c}=0.02$. (b) Above instability threshold; $\alpha_{e}=0.1, \alpha_{c}=0.1$. 


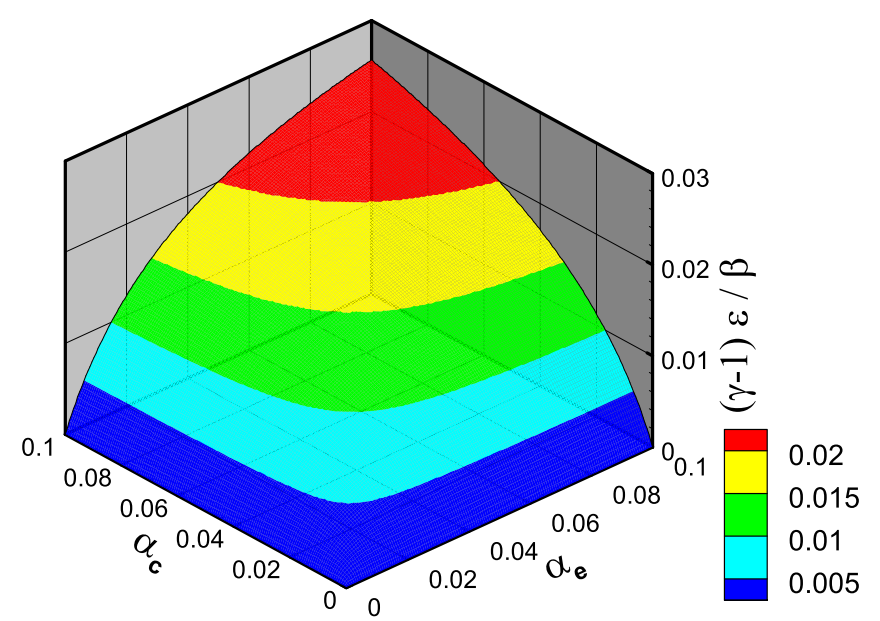

Fig. 3. Instability boundary calculated with the averaging approximation.

times with $r_{0}$, and finding the set of parameters where they coincide. From the comparison with the averaging approximation results (Fig. 4), one may deduce that the instability threshold is well approximated by the averaging approach for small $\alpha_{e}, \alpha_{c}$. This is easy to interpret when recalling that these parameters are the ratios of $\tau$ (that defines the oscillation period) and the respective characteristic times. The averaging method assumes that the system variables change weakly on the time scale $\tau$. This assumption breaks down when either $\alpha_{e}, \alpha_{c}$ or $\varepsilon$ becomes comparable with unity. From numerical solution of the PWL equations it can be found that in general the averaging approximation underestimates the growth rate (cf. Fig. 2(b)). This means that the actual PWL $\varepsilon$ threshold is higher than that predicted by the averaging approximation.

The threshold for the general PWL case cannot be presented as a surface in a 3D space like that of Fig. 3. The threshold $\varepsilon$ value depends in general separately on all four remaining dimensionless parameters. This is evident from Fig. 4 where the threshold value of $(\gamma-1) \varepsilon / \beta$ is shown to depend on both $\beta$ and $\gamma$ when $\alpha_{e}, \alpha_{c}$ become large enough. The usefulness of the averaging approximation is however evident. It gives a lower bound for the instability threshold in a 3D space of the system parameters. Its application can be illustrated on an example of the experimental data

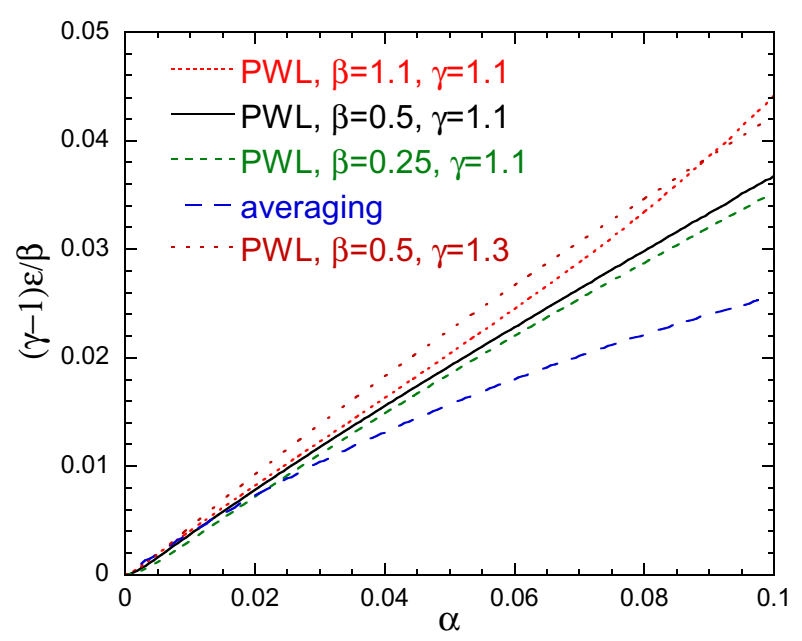

Fig. 4. Instability boundary for the particular case $\alpha_{c}=\alpha_{e} \equiv \alpha$ : comparison of the PWL and averaging approximation results. The averaging approximation curve corresponds to the section of the surface of Fig. 3 by the plane $\alpha_{c}=\alpha_{e}$. presented in Fig. 2(a) of [12]. From the graph one gets the maximum meniscus velocity of $1 \mathrm{~m} / \mathrm{s}$ which corresponds to $v_{R M S}=0.7$ $\mathrm{m} / \mathrm{s}$ from which $\delta_{f}=60 \mu \mathrm{m}$ is obtained with Eq. (1). Note that this value falls within the range $50-100 \mu \mathrm{m}$ obtained by [12] by fitting their experimental data to the FEC model. The coefficients $\alpha_{c}=0.07, \alpha_{e}=0.04, \beta=30, \varepsilon=0.47$ can now be calculated and result in $(\gamma-1) \varepsilon / \beta=10^{-3}$. A comparison with Figs. 3 and 4 shows that the latter value places the model system inside the instability region, in agreement with the experiment, cf. Fig. 2(a) of Das et al. [12] where the oscillations are indeed observed.

\section{Conclusions}

The main result of the above analysis is the criterion for instability (i.e. the startup) of the simplest, single-branch PHP without adiabatic section. It can serve to predict the start-up thresholds of new single branch PHPs (because provides the lower bound for the PHPs with adiabatic section) and for theoretical consideration of the full nonlinear problem (to delimit the region where the oscillations occur). The origin of the self-sustained oscillations is now evident. The evaporation in the evaporator causes the gas pressure increase that propels the meniscus towards the condenser where the condensation causes the pressure decrease and the meniscus returns back to the evaporator. The averaging method is used to solve analytically the piece-wise linear governing equations and to obtain an analytical instability criterion. The analytical analysis of the film evaporation-condensation model was possible thanks to its simplicity. Five dimensionless groups that rule out the oscillation startup are identified; their physical significance is established. The instability region is presented in terms of these groups. Thanks to the averaging approach, the dimensionality of the parametric space, in which the instability boundary is defined, is reduced to 3D. The instability boundary can thus be presented as a surface. The threshold dissipation value obtained from the averaging approximation is the lower bound of its rigorous value that may be obtained only numerically in 5D parametric space.

The model describes correctly the known experimental features of PHP startup: (i) both evaporation and condensation are vital for the oscillation startup, which means that during the oscillation startup the meniscus needs to penetrate into both the condenser and the evaporator, and (ii) the startup of oscillations is controlled by the temperature difference between the evaporator and the condenser. The quantitative comparison is more delicate because of the scarcity of the experimental data for such a system: in spite of the importance of PHP startup issue for applications, the features (i,ii) are about all we know; more experimental data on the PHP startup (in particular, for single branch PHP) are needed.

The above analysis predicts the independence of the startup criterion of the evaporator length under the condition that the oscillation period is kept constant. It can be checked experimentally. Indeed, one can imagine an experiment where both the evaporator length and the liquid plug length are variable so that the constant period constraint may be satisfied.

The theoretical analysis reveals the role of the heat exchange between the gas and the dried out portion of the evaporator walls, which turns out to be the source of energy dissipation in the system. This means that the fluids with low gas heat conductivity are to be chosen for use in the PHP.

\section{Acknowledgements}

The author is grateful to CEA (NTE program) and PSA (PeugeotCitroën) for partial financial support. The financial support of ANR in the framework of the project AARDECO ANR-12-VPTT-005-02 is acknowledged. 


\section{Appendix A. Application of the averaging method}

Let us choose first a ground state that provides a periodical solution. It can be defined by the equations

$\dot{\tilde{x}}=\tilde{v}$

$\dot{\widetilde{T}}=(1-\gamma) \tilde{v}$

$\gamma \dot{\tilde{v}}=\widetilde{T}-\tilde{x}+\tilde{m}$,

$\dot{\tilde{m}}=0$

obtained from the set (21) by putting to zero $\alpha_{i}$ and $\varepsilon$. The dimensionless Eq. (A.1) correspond to Eqs. (18) and (19), the solutions of which are indeed periodical with the unit eigenfrequency,

$\tilde{v}=r \sin \kappa$,

$\tilde{x}=-r \cos \kappa+C$

$\widetilde{T}=(\gamma-1) r \cos \kappa+D$,

$C=D+\tilde{m}$,

where $\kappa=\tilde{t}+\varphi ; r, \varphi, C, D, \tilde{m}$ are arbitrary constants. Therefore, $\tilde{v}, \tilde{x}, \widetilde{T}$ are the "fast" variables. One may now reduce the initial set (21) with the method of variation of arbitrary constants. Assume $r, \varphi, C, D, \tilde{m}$ to be $\tilde{t}$ functions and substitute the expressions (A.2) back into the governing Eqs. (21a-21c). A straightforward reduction results in the equations

$\dot{D}=(1-\gamma) \varepsilon r \cos \kappa-\frac{\varepsilon}{\gamma} D$,

$\dot{r}=\dot{C} \cos \kappa$,

$r \dot{\varphi}=-\dot{C} \sin \kappa$,

which are still rigorous. One may check by solving numerically Eqs. (21d, 21e, A.2d, A.3) that all the variables $r, \varphi, C, D, \widetilde{L}, \tilde{m}$ exhibit small oscillations around slowly varying mean values and are thus the "slow" variables. Their slow variation can be (approximately) determined from equations obtained by averaging Eqs. (21d, 21e, A.3) over the oscillation period $(=2 \pi)$. While averaging the right hand side of the equations, $r, \varphi, C, D, \widetilde{L}, \tilde{m}$ (but not $\dot{C}, \dot{D}, \dot{\tilde{m}}$ ) are to be assumed constant over the period.

From now on, under $r, \varphi, C, D, \widetilde{L}, \tilde{m}$ we mean their averaged counterparts. It is evident that the averaging of Eq. (A.3a) leads to

$\dot{D}=-\frac{\varepsilon}{\gamma} D$

This means that $D \equiv 0$ when $D(\tilde{t}=0)=0$ (which may be assumed without the loss of generality; nonzero $D$ does not impact the conditions of stability because it drops off the remaining equations). Therefore,

$C=\tilde{m}$

Eq. (A.2b) shows that $C$ is the average value of $\tilde{x}$ during a period, while $r$ is its amplitude (Fig. A.5). When $\tilde{x}<0$, the meniscus is located in the evaporator and the value $\tilde{x}=0$ is the boundary between the condenser and the evaporator. Several cases can be distinguished depending on the relation between $|C|$ and $r$. Consider first the case $|C|<r$, where the meniscus penetrates into both condenser and evaporator during one period (Fig. A.5). One may then introduce

$\cos \psi=-\frac{C}{r}$

where $0 \leqslant \psi \leqslant \pi$. The condition $\tilde{x}>0$ (equivalent to $\cos \kappa<C / r$ ) is satisfied when $\pi-\psi<\kappa<\pi+\psi$, see Fig. A.5. The derivative of $\psi$ is

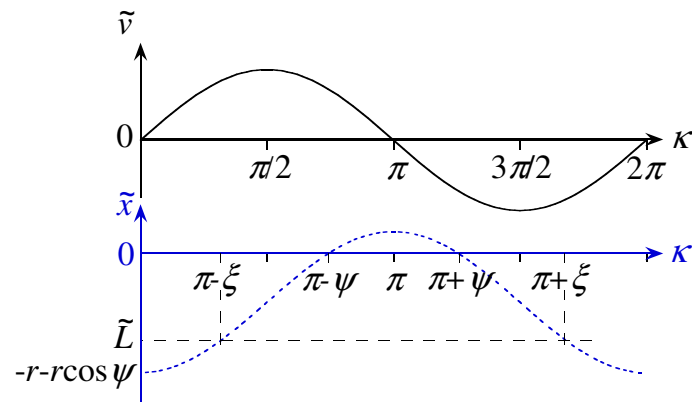

Fig. A.5. Ground state solutions (A.2a) (solid line) and (A.2b) (dotted line) of the nonlinear problem.

$\dot{\psi}=\frac{\dot{\tilde{m}}}{r \sin \psi}+\frac{\dot{r}}{r} \cot \psi$.

Since averaged $\widetilde{L}$ is constant during a period, several cases are possible depending on the relation between $\widetilde{L}, C$ and $r$. Consider first the case where $\widetilde{L}$ is larger than the minimal value of $\tilde{x}$ over the period, i.e. $-r(1+\cos \psi) \leqslant \widetilde{L}<0$, cf. Eqs. (A.2b) and (A.6) and Fig. A.5. This case corresponds to a situation where the condition $\widetilde{L}=\tilde{x}$ may be attained (i.e. the film disappears during some part of the period). The above inequality can be rewritten as $-1 \leqslant \widetilde{L} / r+\cos \psi<\cos \psi$. One may thus introduce a new slowly varying variable $\xi$ (such that $0 \leqslant \xi \leqslant \pi$ ) via

$\widetilde{L}=r(\cos \xi-\cos \psi)$.

This definition means that $\widetilde{L}=\tilde{x}$ when $\kappa=\pi-\xi$ or $\pi+\xi$, cf. Fig. A.5. The derivative of $\xi$ is

$\dot{\xi}=\frac{\dot{\tilde{m}}}{r \sin \xi}-\frac{\dot{\tilde{L}}}{r \sin \xi}+\frac{\dot{r}}{r} \cot \xi$

The averaging of Eqs. (21d), (21e) and (A.3b) results in

$\dot{\tilde{L}}=\frac{1}{2 \pi}\left(\left.\frac{1}{\beta} \int_{0}^{\pi+\xi} \dot{\tilde{m}}\right|_{\alpha_{c} \rightarrow 0} d \kappa+\int_{\pi+\xi}^{2 \pi} \tilde{v} d \kappa\right)$

$=\frac{r}{2 \pi}\left[2 \alpha_{e}(\sin \xi-\sin \psi+\psi \cos \psi-\xi \cos \xi)-(1+\cos \xi)\right]$

$\dot{\tilde{m}}=\frac{\alpha_{e} \beta}{2 \pi}\left(\int_{\pi-\xi}^{\pi-\psi} \tilde{x} d \kappa+\int_{\pi+\psi}^{\pi+\xi} \tilde{x} d \kappa-2 \tilde{\zeta}\right)-\frac{\alpha_{c} \beta}{2 \pi} \int_{\pi-\psi}^{\pi+\psi} \tilde{x} d \kappa$

$=\frac{\beta r}{\pi}\left[\alpha_{e}(\sin \xi-\sin \psi+\psi \cos \psi-\xi \cos \xi)+\alpha_{c}(\psi \cos \psi\right.$

$-\sin \psi)]$

$\dot{r}=\frac{1}{2 \pi}\left[\int_{0}^{2 \pi} \dot{D} \cos \kappa d \kappa-\alpha_{c} \beta \int_{\pi-\psi}^{\pi+\psi} \tilde{x} \cos \kappa d \kappa\right.$

$+\alpha_{e} \beta\left(\int_{\pi-\xi}^{\pi-\psi} \tilde{x} \cos \kappa d \kappa\right.$

$\left.\left.+\int_{\pi+\psi}^{\pi+\xi} \tilde{x} \cos \kappa d \kappa-\widetilde{L} \int_{\pi-\xi}^{\pi+\xi} \cos \kappa d \kappa\right)\right]$

$$
\begin{aligned}
= & -\frac{(\gamma-1) \varepsilon r}{2}+\frac{\beta r}{2 \pi}\left[\alpha_{e}(\psi-\xi-\sin \psi \cos \psi+\sin \xi \cos \xi)\right. \\
& \left.+\alpha_{c}(\psi-\sin \psi \cos \psi)\right] .
\end{aligned}
$$

Similarly, one deduces that the averaging of Eq. (A.3c) results in $\dot{\varphi}=0$. Note that $\dot{\tilde{m}}$ and $\dot{D}$ given by Eqs. (21e) and (A.3a) need to be injected into Eqs. (A.3b) and (A.3c) before averaging them. For clarity, the same notation is kept for the averaged variables. To distinguish 
the averaged and non-averaged values one notes that in Eqs. (A.10), all variables are averaged except of those under integrals. In what follows, all variables are averaged.

The differential equations for $\psi$ and $\xi$ are obtained by the substitution of Eqs. (A.10b), (A.10d) and (A.10f) into Eqs. (A.7) and (A.9), respectively. The resulting two equations form together with Eq. (A.10f) a set of three ODE's for three unknowns $(\psi, \xi, r)$ and can now be solved together. Their initial conditions $\psi=\xi=\pi / 2, r=r_{0}$ and the equality $\varphi=\pi / 2$ correspond to the conditions (23). Note that the right hand sides of the equations for both $\dot{\psi}$ and $\dot{\xi}$ depend only on $\psi, \xi$ and are independent of $r$. This feature will be used below during the stability analysis.

The obtained differential equations are valid until $\xi$ attains $\pi$, which may occur at some time moment $\tilde{t}=t_{\xi}$ when the oscillations are declining. From this moment on, the curves $\tilde{x}(\tilde{t}), \widetilde{L}(\tilde{t})$ do not intersect any more and the introduction of $\xi$ (that defines the point of intersection, cf. Fig. A.5) is not possible any more. The averaged equations for $\tilde{t}>t_{\xi}$ can be obtained from Eqs. (A.10a), (A.10c) and (A.10e) where $\xi$ is now replaced by $\pi$,

$\dot{\tilde{L}}=-\frac{\alpha_{e} r}{\pi}[(\pi-\psi) \cos \psi+\sin \psi+\pi \widetilde{L} / r]$,

$\dot{\tilde{m}}=\frac{\beta r}{\pi}\left\{\alpha_{c}(\psi \cos \psi-\sin \psi)-\alpha_{e}[(\pi-\psi) \cos \psi\right.$

$+\sin \psi+\pi \widetilde{L} / r]\}$

$\dot{r}=-\frac{(\gamma-1) \varepsilon r}{2}+\frac{\beta r}{2 \pi}\left[\alpha_{e}(\psi-\pi-\sin \psi \cos \psi)\right.$

$\left.+\alpha_{c}(\psi-\sin \psi \cos \psi)\right]$.

To close the set of equations, one needs again to use Eq. (A.7). When the oscillations are declining, $\psi$ may in some cases attain zero or $\pi$ at a time $t_{\psi}>t_{\xi}$. The equations that valid for $\tilde{t}>t_{\psi}$ can be readily obtained from Eqs. (A.11) by putting $\psi=0$ or $\psi=\pi$, respectively. They are linear and can be solved analytically. The solutions are however cumbersome and for this reason are not written here.

Note that only Eqs. (A.10) need to be analyzed to study the stability of the system. Those valid for $\tilde{t}>t_{\xi}$ describe only the declining oscillations.

\section{Appendix B. Instability boundary within averaging approximation}

One mentions at once that since both $\psi(\tilde{t})$ and $\xi(\tilde{t})$ functions cannot evolve beyond the interval $(0, \pi)$, they should either come to saturation at $\tilde{t} \rightarrow \infty$ or exhibit an oscillatory behavior. The second option is unlikely within the averaging approach where these functions are slowly varying. Indeed, the numerical calculations show that both $\psi(\tilde{t})$ and $\xi(\tilde{t})$ always saturate when $\tilde{t} \rightarrow \infty$.

Let us first outline the procedure of finding the instability threshold by analyzing the stability of the set of equations for $(\psi, \xi, r)$ resulting from Eqs. (A.7), (A.9) and (A.10). One first obtains the values $\psi(\tilde{t} \rightarrow \infty)$ and $\xi(\tilde{t} \rightarrow \infty)$ by imposing

$\dot{\psi}=0$,

$\dot{\xi}=0$.

As mentioned above, the right hand sides of the differential equations for $\psi$ and $\xi$ depend only on $\psi$ and $\xi$. Thus the conditions (B.1) result in the closed set of two equations that can be solved for $\psi(\tilde{t} \rightarrow \infty)$ and $\xi(\tilde{t} \rightarrow \infty)$. Next, the solutions should be used in the equation

$\dot{r}=0$

that defines the stability threshold at $\tilde{t} \rightarrow \infty$. Indeed, $r$ is the oscillation amplitude and the declining oscillations correspond to $\dot{r}<0$ while the instability condition is $\dot{r}>0$.
We proceed now to the implementation of the outlined procedure. By using Eqs. (A.7) and (A.9) one can rewrite the conditions (B.1) as

$\dot{\tilde{m}}+\dot{r} \cos \psi=0$,

$\dot{\tilde{m}}-\dot{\widetilde{L}}+\dot{r} \cos \xi=0$.

With the account of Eq. (B.2), they reduce to

$\dot{\widetilde{L}}=0$,

$\dot{\tilde{m}}=0$

where the values of the derivatives defined in Eqs. (A.10b) and (A.10d) need to be substituted. After a rearrangement, the obtained equations read

$$
\begin{aligned}
& \cos \xi=2 \alpha_{c}(\sin \psi-\psi \cos \psi)-1, \\
& \alpha_{e}(\sin \xi-\sin \psi+\psi \cos \psi-\xi \cos \xi)=\alpha_{c}(\sin \psi-\psi \cos \psi) .
\end{aligned}
$$

The parameter $\beta$ drops out of Eq. (B.5a) which can easily be solved numerically thus defining the threshold values of $\xi, \psi$ as functions of $\alpha_{c}$ and $\alpha_{e}$. Their solutions can be used in the condition (B.2) equivalent to the equation

$$
\begin{aligned}
& \frac{(\gamma-1) \varepsilon}{\beta}=\frac{1}{\pi}\left[\alpha_{e}(\psi-\xi-\sin \psi \cos \psi+\sin \xi \cos \xi)\right. \\
& \left.+\alpha_{c}(\psi-\sin \psi \cos \psi)\right] .
\end{aligned}
$$

Eq. (B.5b) defines the instability threshold. It expresses $(\gamma-1) \varepsilon / \beta$ as a function of $\alpha_{c}$ and $\alpha_{e}$.

\section{References}

[1] H. Akachi, Structure of micro-heat pipe, US Patent 5219020, 1993.

[2] Y. Zhang, A. Faghri, Advances and unsolved issues in pulsating heat pipes, Heat Transfer Eng. 29 (1) (2008) 20-44, http://dx.doi.org/10.1080/ 01457630701677114.

[3] S. Khandekar, P.K. Panigrahi, F. Lefèvre, J. Bonjour, Local hydrodynamics of flow in a pulsating heat pipe: a review, Front. Heat Pipes 1 (2) (2010) 023003 http://dx.doi.org/10.5098/fhp.v1.2.3003.

[4] S. Lips, A. Bensalem, Y. Bertin, V. Ayel, C. Romestant, J. Bonjour, Experimental evidences of distinct heat transfer regimes in pulsating heat pipes (PHP), Appl. Thermal Eng. 30 (8-9) (2010) 900-907, http://dx.doi.org/10.1016/ j.applthermaleng.2009.12.020. ISSN 1359-4311.

[5] F.P. Bretherton, The motion of long bubbles in tubes, J. Fluid Mech. 10 (1961) 166-188, http://dx.doi.org/10.1017/S0022112061000160.

[6] P. Aussillous, D. Quéré, Quick deposition of a fluid on the wall of a tube, Phys. Fluids 12 (10) (2000) 2367-2371, http://dx.doi.org/10.1063/1.1289396.

[7] L.D. Landau, B.V. Levich, Dragging of a liquid by a moving plate, Acta Physicochim. USSR 17 (1942) 42-54.

[8] G. Lagubeau, Propulsion par moteur pop-pop, Master's thesis, supervisor: D. Quéré, PMMH-ESPCI, Paris, 2006.

[9] M.B. Shafii, A. Faghri, Y. Zhang, Thermal modeling of unlooped and looped pulsating heat pipes, J. Heat Transfer 123 (6) (2001) 1159-1172, http:// dx.doi.org/10.1115/1.1409266.

[10] Y. Zhang, A. Faghri, Heat transfer in a pulsating heat pipe with open end, Int. J. Heat Mass Transfer 45 (4) (2002) 755-764, http://dx.doi.org/10.1016/S00179310(01)00203-4.

[11] R.T. Dobson, Theoretical and experimental modelling of an open oscillatory heat pipe including gravity, Int. J. Thermal Sci. 43 (2) (2004) 113-119, http:// dx.doi.org/10.1016/j.ijthermalsci.2003.05.003.

[12] S.P. Das, V.S. Nikolayev, F. Lefèvre, B. Pottier, S. Khandekar, J. Bonjour, Thermally induced two-phase oscillating flow inside a capillary tube, Int. J. Heat Mass Transfer 53 (19-20) (2010) 3905-3913, http://dx.doi.org/10.1016/ j.ijheatmasstransfer.2010.05.009. ISSN 0017-9310.

[13] M. Rao, F. Lefèvre, J. Bonjour, S. Khandekar, Thermally induced two-phase oscillating flow in a capillary tube: theoretical and experimental investigations, in: Proceedings of 16th International Heat Pipe Conference, INSA Lyon, Lyon, France, 2012

[14] F. Bonnet, P. Gully, V. Nikolayev, Experimental study of a single branch cryogenic pulsating heat pipe: first results, in: Proceedings Eurotherm sem. on gravitational effects on liquid-vapor phase change, IUSTI, Hyeres, France, 2011

[15] V.S. Nikolayev, A dynamic film model of the pulsating heat pipe, J. Heat Transfer 133 (8) (2011) 081504, http://dx.doi.org/10.1115/1.4003759.

[16] V.S. Nikolayev, Towards predictive modeling of pulsating heat pipes, in: Proceedings of 16 th International Heat Pipe Conference, INSA Lyon, Lyon, France, 2012. 
[17] V.S. Nikolayev, Dynamics of the triple contact line on a nonisothermal heate at partial wetting, Phys. Fluids 22 (8) (2010) 082105, http://dx.doi.org/ 10.1063/1.3483558.

[18] S.K. Wilson, S.H. Davis, S.G. Bankoff, The unsteady expansion and contraction of a long two-dimensional vapour bubble between superheated or subcooled parallel plates, J. Fluid Mech. 391 (1999) 1-27, http://dx.doi.org/10.1017/ S0022112099004516
[19] V.S. Nikolayev, Comment on "Flow and heat transfer of liquid plug and neighboring vapor slugs in a pulsating heat pipe" by Yuan, Qu, \& Ma, Int. J. Heat Mass Transfer 54 (9-10) (2011b) 2226-2227. <http://dx.doi.org/10.1016/ j.ijheatmasstransfer.2011.01.007>.

[20] A. Nayfeh, Introduction to Perturbation Techniques, Wiley-VCH, Weinheim, 2004. ISBN 9783527618453. 